\title{
On the extra phase correction to the semiclassical spin coherent-state propagator
}

\author{
Mikhail Pletyukhov* \\ Institut für Theoretische Festkörperphysik, \\ Universität Karlsruhe, D-76128 Karlsruhe, Germany and \\ Institut für Theoretische Physik, Universität Regensburg, D-93040 Regensburg, Germany
}

\begin{abstract}
The problem of an origin of the Solari-Kochetov extra-phase contribution to the naive semiclassical form of a generalized phase-space propagator is addressed with the special reference to the $\mathrm{su}(2)$ spin case which is the most important in applications. While the extra-phase correction to a flat phase-space propagator can straightforwardly be shown to appear as a difference between the principal and the Weyl symbols of a Hamiltonian in the next-to-leading order expansion in the semiclassical parameter, the same statement for the semiclassical spin coherent-state propagator holds provided the Holstein-Primakoff representation of the su(2) algebra generators is employed.
\end{abstract}

*Electronic address: pletmikh@tfp.physik.uni-karlsruhe.de 


\section{INTRODUCTION}

The spin coherent-state path integral appears to be very useful in many physical problems that involve quantum $\mathrm{su}(2)$ spins. In particular, one of its most significant practical applications is the study of spin tunneling in the semiclassical limit [1]-[4]. However, as it was remarked in [5], the straightforward computation of the semiclassical propagator [6] or the tunnel splitting 7] yields results that are incorrect beyond the leading semiclassical order. Examples of other systems where the large spin limit gives a good qualitative picture, while the first quantum correction is either ignored or fixed by heuristic considerations, can be found in [8].

Recently, Stone et al [5]. have restored the reliability of the semiclassical expression for the spin coherent-state propagator, thus effectively rehabilitating the use of the continuous-time spin coherent-state path integral. The crucial point in that approach is the recognition of an importance and the explanation of the origin of a previously discovered quantum correction [9]-[11] to the naive form of the semiclassical coherent-state propagator. It has been pointed out that the functional determinant arising from the evaluation of the functional integral about the classical path possesses a U(1) gauge anomaly, its proper regularization resulting in the extra-phase contribution. Originally Solari [9] obtained this extra-phase correction through a careful calculation of the path integral in the discrete-time approximation. Kochetov [10] derived it independently considering the continuous-time version of the spin coherent-state path integral in the semiclassical limit. A discrete-time evaluation similar to that of Solari was carried out by Vieira and Sacramento 11] who have also reproduced the same result.

The relevance of the Solari-Kochetov phase has been justified in the application of the spin coherent-state path integral to the calculation of the tunnel splitting of the classically degenerate ground state for a family of models that includes a realistic approximation to the molecular magnet $\mathrm{Fe}_{8}[8]$. It has been also noticed in [12] that the modification of the Gutzwiller trace formula [13] for systems with a coupling of the translational and spin degrees of freedom should also contain this extra phase in the combined limit $\hbar \rightarrow 0, S \rightarrow \infty$, $\hbar S=$ const.

In this paper we offer a point of view on the origin of the Solari-Kochetov phase, which complements the analysis made in [5]. 
To establish our notations we briefly outline in Sec. II some basic facts concerning quantization on cotangent bundles and coadjoint group orbits, the phase space manifolds that are most frequently encountered in applications. In Sec. III we consider in detail semiclassical propagators on flat phase spaces. We observe that the arising extra phase can be interpreted as a difference between the principal and the Weyl symbols of a Hamiltonian in the next-to-leading order in $\hbar$. On the other hand, various symbols, or various quantization schemes, are closely related to the operator ordering procedures. One can then alternatively state that the extra phase is the difference between the naive classical Hamiltonian and the expectation value of the Weyl-ordered operator [14]. Obviously, both formulations are equivalent, though the former provides a more efficient way to compute the extra phases, since it is based on the Wigner-Weyl calculus and implies an extensive use of the Moyal formula [15].

In Sec. IV we discuss the semiclassical spin coherent-state propagator and its specific ingredient — the Solari-Kochetov phase. We also briefly outline the relation between the covariant and contravariant quantization schemes which are usually employed for the path integral construction in the spin case.

To obtain the Solari-Kochetov phase using the analogy with the flat case we, in the first place, need a proper definition of a Weyl symbol of the quantum spin Hamiltonian. The point is that a standard Weyl quantization is well-defined on a classical phase space $M$, provided it appears as a cotangent bundle to a certain configuration space $Q, M=T^{*} Q$. While $Q$ may in principle be a compact manifold, $T^{*} Q$ is always noncompact. A classical phase space of a spin is, however, a compact finite volume manifold, the two-sphere $S^{2}$, which is not a cotangent bundle. Therefore, there does not seem to exist any natural global definition of appropriate Weyl symbols of the $\mathrm{su}(2)$ spin operators.

To partly circumvent this apparent difficulty, we employ in Sec. $\nabla$ a special HolsteinPrimakoff representation of the $\mathrm{su}(2)$ algebra [16]. It expresses the $\mathrm{su}(2)$ generators in terms of $\hat{a}, \hat{a}^{\dagger}, \hat{a}^{\dagger} \hat{a}$ - the standard generators of the Heisenberg-Weyl algebra, and allows for a formal application of the standard Wigner-Weyl calculus to spin Hamiltonians. We then are able to define the Weyl symbols of the spin operators, provided the semiclassical representations of $\mathrm{su}(2)$ are considered, and to determine their difference from the components of the classical spin. This difference appears to be well-defined in the semiclassical limit $S \rightarrow \infty$, exactly reproducing the Solari-Kochetov phase. 


\section{PHASE-SPACE QUANTIZATION}

The classical phase space can be thought of as a pair $(M, w)$ where $M$ is a $2 n$-dimensional Riemannian manifold and $w$ stands for a closed non-degenerate symplectic two-form on it. A group of canonical transformations $G$ acting on $M$ transitively leaves $w$ invariant. Quantization on $M$ amounts to constructing a complex line bundle over $M$ with a connection one-form $\theta$ such that $d \theta=w$. A quantum Hilbert space is then constructed out of sections of the bundle. When lifted to the bundle, the action of $G$ gives rise to a unitary but reducible representation of $G$. In order to select an irreducible component, the action of $G$ is restricted to the subspace of those sections which are covariantly constant along $n$ linearly independent vector fields $\chi_{1}, \ldots, \chi_{n}$ on $M$ such that $w\left(\chi_{i}, \chi_{j}\right)=0$. This procedure is usually referred to as fixing of the polarization [17].

To illustrate these definitions we briefly discuss two examples of phase spaces, which appear to be our main concern here, cotangent bundles and coadjoint group orbits.

Local coordinates on a cotangent bundle $M=T^{*} Q$ can always be separated into "coordinates", $q^{j}$, and "momenta", $p_{j}$, which are canonically conjugated to the $q^{j}$, with the globally defined canonical symplectic one-form $\theta=(1 / \hbar)\left(p^{j} d q_{j}-q^{j} d p_{j}\right)$. Covariantly constant sections $f(q, p)$ along the vector field $\chi=\partial_{p}$ (we put for simplicity $n=1$ ) are then defined by

$$
\nabla_{p} f:=\left(\partial_{p}+i \theta_{p}\right) f=0
$$

whence $f(q, p) \sim e^{i p q / \hbar} \psi(q)$. The quantum Hilbert space appears then as the space of squareintegrable functions $\psi(q)$. When considering the time evolution, one usually picks up initial and final states as the eigenstates of the operator $\hat{q}, \hat{q}\left|q_{0}\right\rangle=q_{0}\left|q_{0}\right\rangle$, which corresponds to the choice $\psi(q) \sim \delta\left(q-q_{0}\right)$. Analogously, the choice $\chi=\partial_{q}$ results in quantization in terms of the $p$-dependent sections, $f(q, p) \sim e^{-i p q / \hbar} \psi(p)$. In the following we will be considering quantum propagators on $T^{*} Q$ having the form

$$
K\left(p_{f}, q_{i}, T\right):=\left\langle p_{f}\left|e^{-i \hat{H} T / \hbar}\right| q_{i}\right\rangle
$$

where initial and final quantum states belong to different polarizations.

Formally, the Weyl symbol of the operator $\hat{H}(\hat{q}, \hat{p})$ is given by

$$
H_{W}(q, p)=\int_{Q} d x \sqrt{g} e^{i p x / \hbar}\langle q-x / 2|\hat{H}| q+x / 2\rangle
$$


where $g$ denotes the determinant of a restriction of a full metric tensor onto $Q$. As already said, the separation of the local coordinates into $(q, p)$ is defined on a cotangent bundle globally (because $\theta$ exists globally). Accordingly, the symbol $H_{W}(q, p)$ is defined on the phase space manifold $T^{*} Q$ globally as well.

The second important class of classical phase spaces is the so-called coadjoint orbits of Lie groups [17]. These symplectic $G$-homogeneous manifolds appear as natural classical phase spaces in the instances where quantum Hamiltonians allow for representations in terms of the Lie group generators. For example, the quantum spin dynamics is governed by a Hamiltonian built out of the su(2) generators. Spin classical phase space appears then as an $\mathrm{SU}(2)$ orbit - the two-sphere $S^{2}[18$ ], which is a compact curved manifold. It can be covered by two local charts with complex coordinates $(z, \bar{z})$ and $(w, \bar{w})$ defined by projections from the North and South Poles, respectively.

Since there is no globally defined symplectic one-form on $S^{2}$, it does not admit a global separation of coordinates into $q$ 's and $p$ 's. Instead, the locally defined $\mathrm{su}(2)$ symplectic one-forms (in the representation with spin $S) \theta_{1}=i S(\bar{z} d z-z d \bar{z}) /\left(1+|z|^{2}\right)$ and $\theta_{2}=$ $i S(\bar{w} d w-w d \bar{w}) /\left(1+|w|^{2}\right)$, where $w=1 / z$, are related in the charts' overlap by a $\mathrm{U}(1)$ gauge transformation, $\theta_{1}=\theta_{2}+i S d \log (z / \bar{z})$.

The covariantly constant section of the monopole $P\left(S^{2}, U(1)\right)$ bundle that forms a quantum Hilbert space for spin can be chosen as an $\mathrm{su}(2)$ coherent state. In particular,

$$
\nabla_{\bar{z}}|z\rangle_{1}:=\left(\partial_{\bar{z}}+i \theta_{1 \bar{z}}\right)|z\rangle_{1}=0,
$$

where $|z\rangle_{1}$ denotes the $\operatorname{su}(2)$ coherent state in the local chart $(z, \bar{z})$. The $\operatorname{su}(2)$ quantum phase-space propagator can then be written down in the form

$$
K\left(\bar{z}_{f}, z_{i}, T\right):={ }_{1}\left\langle z_{f}\left|e^{-i \hat{H} T / \hbar}\right| z_{i}\right\rangle_{1},
$$

where the initial and final states belong to different polarizations.

Given a spin quantum Hamiltonian $\hat{H}$, its classical symbol may be conveniently chosen as an expectation value in the coherent state, the so-called covariant symbol of the spindependent operator, $H_{1}^{\text {cov }}(\bar{z}, z)={ }_{1}\langle z|\hat{H}| z\rangle_{1}$. In spite of its appearance, this symbol is defined globally on $S^{2}$ : the $P\left(S^{2}, U(1)\right)$ local sections are related in the charts' overlap by the U(1) gauge transformation

$$
{ }_{2}\langle 1 / z \mid z\rangle_{1}=(z / \bar{z})^{S} \in U(1) .
$$


Therefore, $H_{1}^{\operatorname{cov}}(\bar{z}, z)=H_{2}^{\operatorname{cov}}(\bar{w}, w)$ for $w=1 / z$.

Concluding the section, we may state: though it might be in principle possible to construct a Weyl symbol for the su(2) spin locally on $S^{2}$, there seems to be no way to extend such a definition globally to the whole sphere.

\section{FLAT PHASE-SPACE PROPAGATOR}

The well-known van Vleck representation for the semiclassical propagator on a flat configuration space [19] has its counterpart in the phase space $T^{*} R^{2}$ which is the semiclassical approximation for the propagator (2)

$$
K\left(p_{f}, q_{i}, T\right)=\frac{1}{\sqrt{2 \pi \hbar}}\left(-\frac{\partial^{2} \mathcal{R}_{c l}}{\partial p_{f} \partial q_{i}}\right)^{1 / 2} e^{i \mathcal{R}_{c l}\left(p_{f}, q_{i}, T\right) / \hbar}
$$

where

$$
\mathcal{R}_{c l}\left(p_{f}, q_{i}, T\right)=-\frac{1}{2}\left(p_{f} q_{c l}(T)+p_{c l}(0) q_{i}\right)+\int_{0}^{T} d t\left(-\frac{1}{2}\left(\dot{p}_{c l} q_{c l}-p_{c l} \dot{q}_{c l}\right)-H\left(q_{c l}, p_{c l}\right)\right) .
$$

The classical dynamics of the system is governed by the principal symbol $H(q, p)$ of the quantum Hamiltonian $\hat{H}$ :

$$
\begin{array}{ll}
\dot{q}=\frac{\partial H(q, p)}{\partial p}, & q(0)=q_{i}, \\
\dot{p}=-\frac{\partial H(q, p)}{\partial q}, & p(T)=p_{f} .
\end{array}
$$

We would also call $H(q, p)$ the naive classical Hamiltonian since it can be obtained by just "dropping hats" in $\hat{H}$.

Formally, the principal symbol $H(q, p)$ is obtained in the limit $\hbar \rightarrow 0$ of the Weyl symbol (3). The Weyl symbol of the product of two operators is given by the Moyal product [15] of two respective symbols. The Moyal formula can be written in the symbolic form

$$
(\hat{F} \hat{G})_{W}=F_{W} * G_{W}=F_{W} e^{i \hbar \mathcal{L} / 2} G_{W}
$$

where the operator

$$
\mathcal{L}=\frac{\overleftarrow{\partial}}{\partial q} \frac{\vec{\partial}}{\partial p}-\overleftarrow{\frac{\partial}{\partial p}} \frac{\vec{\partial}}{\partial q}
$$

is associated with the Poisson bracket

$$
F_{W} \mathcal{L} G_{W}=\left\{F_{W}, G_{W}\right\}_{q, p}=\frac{\partial F_{W}}{\partial q} \frac{\partial G_{W}}{\partial p}-\frac{\partial F_{W}}{\partial p} \frac{\partial G_{W}}{\partial q} .
$$


Expanding (11) into a series of $\hbar$, we obtain the leading and the next-to-leading contributions to the Moyal formula

$$
(\hat{F} \hat{G})_{W}=F_{W} G_{W}+\frac{i \hbar}{2}\left\{F_{W}, G_{W}\right\}_{q, p}+O\left(\hbar^{2}\right)
$$

Respectively, the leading contribution to the Weyl symbol of the commutator

$$
([\hat{F}, \hat{G}])_{W}=(\hat{F} \hat{G}-\hat{G} \hat{F})_{W}=F_{W} * G_{W}-G_{W} * F_{W}=i \hbar\left\{F_{W}, G_{W}\right\}_{q, p}+O\left(\hbar^{3}\right)
$$

establishes the "correspondence principle" between commutators and Poisson brackets.

However, the expression (17) is, in general, incorrect. Besides the Morse index that accounts for the number of conjugate points [20], there may appear another discrepancy between the representation of (17) and that of a correct asymptotic propagator. It happens when the quantum Hamiltonian contains the terms that mix $\hat{q}$ and $\hat{p}$. Unless $\hat{H}$ is Weyl (symmetrically) ordered, it is not sufficient to take into account just the principal symbol $H(q, p)$ for the calculation of $\mathcal{R}_{c l}$.

Thus, we make here the following proposition: in the semiclassical evaluation of the propagator of type (7) it is necessary to use the Weyl symbol of the quantum Hamiltonian:

$$
H_{W}(q, p)=H(q, p)-\delta H(q, p)+O\left(\hbar^{2}\right), \quad \delta H(q, p) \sim O(\hbar)
$$

Though the classical dynamics is governed by the principal symbol, or naive classical Hamiltonian, $H(q, p)$, the next order term $\delta H(q, p)$ divided by $\hbar$, nevertheless, contributes to the phase of the semiclassical propagator. If the quantum Hamiltonian is a polynomial in $\hat{q}$ and $\hat{p}$, it is easy to calculate $\delta H(q, p)$ using the Moyal formula (14) and the obvious fact that $(\hat{q})_{W}=q$ and $(\hat{p})_{W}=p$.

Let us introduce the complex variables

$$
\alpha=\frac{1}{\sqrt{2}}(q+i p), \quad \bar{\alpha}=\frac{1}{\sqrt{2}}(q-i p) .
$$

They are normalized to be the Weyl symbols of the operators

$$
\hat{A}=\frac{1}{\sqrt{2}}(\hat{q}+i \hat{p}), \quad \hat{A}^{\dagger}=\frac{1}{\sqrt{2}}(\hat{q}-i \hat{p})
$$

which satisfy the commutation relation

$$
\left[\hat{A}, \hat{A}^{\dagger}\right]=\hbar
$$


We can define the Weyl symbols $F_{W}(\bar{\alpha}, \alpha)$ and $G_{W}(\bar{\alpha}, \alpha)$ and the Poisson bracket

$$
\left\{F_{W}(\bar{\alpha}, \alpha), G_{W}(\bar{\alpha}, \alpha)\right\}_{\bar{\alpha}, \alpha}=i\left(\frac{\partial F_{W}}{\partial \bar{\alpha}} \frac{\partial G_{W}}{\partial \alpha}-\frac{\partial F_{W}}{\partial \alpha} \frac{\partial G_{W}}{\partial \bar{\alpha}}\right)
$$

by making a change of variables (17) in $F_{W}(q, p)$ and $G_{W}(q, p)$ and in the Poisson bracket (13), respectively. In turn, after the transformation (18) the operator which is Weyl-ordered in $\hat{A}, \hat{A}^{\dagger}$ converts into the operator Weyl-ordered in $\hat{q}, \hat{p}$. Note also that for the Weyl-ordered operator (either in $\hat{A}, \hat{A}^{\dagger}$ or in $\hat{q}, \hat{p}$ ), its Weyl symbol yields the principal symbol, the higher order terms being identically zero. This justifies the above definition of the Weyl symbols $F_{W}(\bar{\alpha}, \alpha)$ and $G_{W}(\bar{\alpha}, \alpha)$.

Taking into account a definite correspondence between the symbols and the ordering procedures, we can therefore interpret the emergence of the extra phase $\delta H / \hbar$ as an artifact of the operator ordering.

For a more detailed explanation, we would like to discuss the results of [21]. In particular, there has been established the relation between semiclassical results for a propagator obtained within different quantization schemes. The " $\lambda$-quantization" infers the choice of the $\lambda$-symbol [22]

$$
H^{(\lambda)}(\bar{\alpha}, \alpha)=\operatorname{Tr}\left[\hat{H} \hat{R}_{\lambda}(\bar{\alpha}, \alpha)\right], \quad \lambda \in[0,1]
$$

where

$$
\hat{R}_{\lambda}(\bar{\alpha}, \alpha)=\frac{1}{\pi \hbar} \int d^{2} \xi e^{-(1-2 \lambda) \bar{\xi} \xi / 2 \hbar} e^{\left\{\xi\left(\bar{\alpha}-\hat{A}^{\dagger}\right)-\bar{\xi}(\alpha-\hat{A})\right\} / \hbar} .
$$

The particular cases of $\lambda=0,1 / 2,1$ correspond to the covariant (coherent-state), Weyl and contravariant symbols, respectively. The arbitrary $\lambda$-symbol is linked to the covariant symbol through the relation

$$
H^{(0)}(\bar{\alpha}, \alpha)=\left(\hat{T}_{\lambda}(\Delta) H^{(\lambda)}\right)(\bar{\alpha}, \alpha), \quad \hat{T}_{\lambda}(\Delta)=e^{\hbar \lambda \Delta}
$$

where $\Delta=\partial^{2} / \partial \alpha \partial \bar{\alpha}$ is the Laplace-Beltrami operator on the complex plane.

In the semiclassical limit $\hbar \rightarrow 0$ the expression (23) can be expanded as

$$
H^{(0)}=H^{(\lambda)}+\hbar \lambda \Delta H^{(\lambda)}+O\left(\hbar^{2}\right) .
$$

Therefore, for arbitrary $\lambda, \lambda^{\prime} \in[0,1]$ we have

$$
H^{\left(\lambda^{\prime}\right)}=H^{(\lambda)}-\hbar\left(\lambda^{\prime}-\lambda\right) \Delta H^{(\lambda)}+O\left(\hbar^{2}\right)
$$


The expression for the semiclassical propagator reads [21]

$$
K_{\text {scl }}^{\text {flat }}=\left(i \frac{\partial^{2} \mathcal{R}_{c l}^{(\lambda)}}{\partial \bar{\alpha}_{f} \partial \alpha_{i}}\right)^{1 / 2} \exp \left\{i \frac{\mathcal{R}_{c l}^{(\lambda)}}{\hbar}+i\left(\frac{1}{2}-\lambda\right) \int_{0}^{T} B^{(\lambda)} d t\right\}
$$

where

$$
\begin{aligned}
\mathcal{R}^{(\lambda)}\left(\bar{\alpha}_{f}, \alpha_{i}, T\right) & =-\frac{i}{2}\left(\bar{\alpha}_{f} \alpha_{c l}(T)+\bar{\alpha}_{c l}(0) \alpha_{i}-\left|\alpha_{f}\right|^{2}-\left|\alpha_{i}\right|^{2}\right) \\
& +\int_{0}^{T} d t\left(-\frac{i}{2}\left(\dot{\bar{\alpha}}_{c l} \alpha_{c l}-\bar{\alpha}_{c l} \dot{\alpha}_{c l}\right)-H^{(\lambda)}\left(\bar{\alpha}_{c l}, \alpha_{c l}\right)\right)
\end{aligned}
$$

and

$$
B^{(\lambda)}=\Delta H^{(\lambda)}=\Delta H^{(1 / 2)}+O(\hbar)=\Delta H^{(0)}+O(\hbar) .
$$

The terms of the order $O(\hbar)$ in $B^{(\lambda)}$ as well as the dependence of the prefactor on $\lambda$ are inessential due to the very structure of the asymptotic expression (26) $). O(\hbar)$-terms are also negligible in the classical equations of motion:

$$
\begin{array}{ll}
\dot{\alpha}=-i \frac{\partial H^{(\lambda)}}{\partial \bar{\alpha}}+O(\hbar), & \alpha(0)=\alpha_{i}, \\
\dot{\bar{\alpha}}=i \frac{\partial H^{(\lambda)}}{\partial \alpha}+O(\hbar), & \bar{\alpha}(T)=\bar{\alpha}_{f} .
\end{array}
$$

Note that for $\lambda=1 / 2$ (Weyl quantization) the $B$-term drops out from (26). Since the semiclassical propagator should not depend on $\lambda$, i.e. on the choice of the quantization scheme, the extra-phase correction just compensates for the difference between $\lambda$ and Weyl symbols in the next-to-leading order in $\hbar$ [see (25) for $\lambda^{\prime}=1 / 2$ ].

Suppose that the quantum Hamiltonian $\hat{H}$ belongs to a family of specifically ordered Hamiltonians [23], also parametrized by $\lambda \in[0,1]$,

$$
\hat{H}_{\lambda}\left(\hat{A}^{\dagger}, \hat{A}\right)=\frac{1}{(\pi \hbar)^{2}} \int d^{2} \alpha d^{2} \beta H(\bar{\alpha}, \alpha) e^{(1-2 \lambda) \bar{\beta} \beta / 2 \hbar} e^{\left\{\beta\left(\bar{\alpha}-\hat{A}^{\dagger}\right)-\bar{\beta}(\alpha-\hat{A})\right\} / \hbar},
$$

where the particular cases of $\lambda=0,1 / 2,1$ correspond to the normal, Weyl and antinormal orderings, respectively. One can establish the one-to-one correspondence between the operators (31) and the symbols (21). It follows from the observation that the $\lambda$-symbol of the $\lambda$-ordered operator yields the principal symbol $H(\bar{\alpha}, \alpha)$. Thus, the result (26) of [21] actually proves our proposition for the Hamiltonians (31).

The problem of the extra-phase contribution was often encountered in the coherent-state semiclassics based on the covariant quantization scheme. We can illustrate it with the following example. 
Let us consider the normally ordered operator

$$
\hat{H}_{\lambda=0}=\sum_{m, n} h_{m n} \hat{A}^{\dagger m} \hat{A}^{n}
$$

Its covariant (coherent-state) symbol

$$
H^{(0)}(\bar{\alpha}, \alpha)=\left\langle\alpha\left|\hat{H}_{0}\right| \alpha\right\rangle=\sum_{m, n} h_{m n} \bar{\alpha}^{m} \alpha^{n}
$$

is found from (21) with $\lambda=0$, or, equivalently, by taking an expectation value in the Heisenberg-Weyl coherent state

$$
|\alpha\rangle=e^{-\bar{\alpha} \alpha / 2 \hbar} e^{\alpha \hat{A}^{\dagger} / \hbar}|0\rangle, \quad\langle\alpha \mid \alpha\rangle=1
$$

Obviously, the covariant symbol (33) coincides with the principal symbol.

The Weyl symbol of (32) is

$$
H^{(1 / 2)}=\sum_{m, n} h_{m n} \bar{\alpha}^{m} \alpha^{n}+\frac{i \hbar}{2} \sum_{m, n} h_{m n}\left\{\bar{\alpha}^{m}, \alpha^{n}\right\}_{\bar{\alpha}, \alpha}+O\left(\hbar^{2}\right)=H^{(0)}-\frac{\hbar}{2} \Delta H^{(0)}+O\left(\hbar^{2}\right) .
$$

Thus, we can deduce that

$$
\frac{\delta H}{\hbar}=\frac{1}{2} \Delta H^{(0)}
$$

The same expression for the phase correction has been derived in Ref. [24], where the WKB approximation in the Bargmann representation has been considered. In the case $h_{11}=\omega$ and $h_{m n}=0$ for $m, n \neq 1$, we see that $\delta H / \hbar=\omega / 2$. When multiplied by $T$, it exactly coincides with the required phase correction to the semiclassical coherent-state propagator of the harmonic oscillator. Its inclusion is sometimes referred to as a restoration of zero-point energy in the first order of $\hbar$.

\section{SEMICLASSICAL SPIN COHERENT-STATE PROPAGATOR}

A quantum spin Hamiltonian $\hat{H}=\hat{H}(\hat{\mathbf{s}})$ is a function of spin algebra generators which satisfy the $\mathrm{su}(2)$ commutation relations

$$
\left[\hat{s}_{+}, \hat{s}_{-}\right]=2 \hat{s}_{3}, \quad\left[\hat{s}_{3}, \hat{s}_{ \pm}\right]= \pm \hat{s}_{ \pm}
$$

where $\hat{s}_{ \pm}=\hat{s}_{1} \pm i \hat{s}_{2}$. 
There actually exist two quantization schemes for spin [25] — covariant and contravariant - that are usually employed for the path-integral construction and its further semiclassical approximations.

The covariant quantization scheme is based on the coherent-state representation of the quantum Hamiltonian: the covariant symbol is defined as an expectation value

$$
H^{\operatorname{cov}}(\bar{z}, z) \equiv H(\bar{z}, z)={ }_{1}\langle z|\hat{H}| z\rangle_{1}
$$

in the spin coherent state

$$
|z\rangle_{1}=(1+\bar{z} z)^{-S} e^{z \hat{s}_{+}}|S,-S\rangle, \quad{ }_{1}\langle z \mid z\rangle_{1}=1
$$

where $|S,-S\rangle$ is the lowest spin state in the $2 S+1$-dimensional representation of $\mathrm{SU}(2)$. The second family of the spin coherent states is given by

$$
|w\rangle_{2}=(1+\bar{w} w)^{-S} e^{w \hat{s}_{-}}|S, S\rangle, \quad{ }_{2}\langle w \mid w\rangle_{2}=1
$$

$|S, S\rangle$ being the highest spin state.

In the sequel, all spin coherent states will be drawn from the first family, $|z\rangle:=|z\rangle_{1}$.

The spin coherent-state propagator (5) can be approximated in the semiclassical limit $S \rightarrow \infty$ by

$$
K_{s c l}\left(\bar{z}_{f}, z_{i}, T\right)=\left(i \frac{\left(1+\bar{z}_{f} z_{c l}(T)\right)\left(1+\bar{z}_{c l}(0) z_{i}\right)}{2 S} \frac{\partial^{2} \mathcal{R}_{c l}}{\partial z_{i} \partial \bar{z}_{f}}\right)^{1 / 2} e^{i \mathcal{R}_{c l}\left(\bar{z}_{f}, z_{i}, T\right)+\frac{i}{2} \int_{0}^{T} \phi_{S K}(t) d t} .
$$

The validity of this formula has been proven in [5]. It has been also shown that the degree of its accuracy, assuming errors of at most $O(1 / S)$, is uniform in $T$.

The leading contribution to the phase of $K_{s c l}$ is the classical action

$$
\begin{aligned}
\mathcal{R}_{c l}\left(\bar{z}_{f}, z_{i}, T\right) & =-i S\left\{\ln \left[\left(1+\bar{z}_{f} z_{c l}(T)\right)\left(1+\bar{z}_{c l}(0) z_{i}\right)\right]-\ln \left[\left(1+\left|z_{f}\right|^{2}\right)\left(1+\left|z_{i}\right|^{2}\right)\right]\right\} \\
& +\int_{0}^{T}\left\{-i S \frac{\dot{\bar{z}}_{c l} z_{c l}-\bar{z}_{c l} \dot{z}_{c l}}{1+\bar{z}_{c l} z_{c l}}-H\left(\bar{z}_{c l}, z_{c l}\right)\right\} d t .
\end{aligned}
$$

(Note the distinction up to a factor of $i$ in our notation and that of [5] as well as the difference in the normalization of the spin coherent states.) Classical trajectories $z_{c l}(t), \bar{z}_{c l}(t)$ are to be found from the classical equations of motion

$$
\begin{array}{ll}
\dot{z}=-i \frac{(1+\bar{z} z)^{2}}{2 S} \frac{\partial H}{\partial \bar{z}}, & z(0)=z_{i}, \\
\dot{\bar{z}}=i \frac{(1+\bar{z} z)^{2}}{2 S} \frac{\partial H}{\partial z}, & \bar{z}(T)=z_{f} .
\end{array}
$$


The Solari-Kochetov phase, or the first quantum phase correction to $K_{s c l}$, is expressed through

$$
\phi_{S K}(t)=\left.\frac{1}{2}\left(\frac{\partial}{\partial \bar{z}} \frac{(1+\bar{z} z)^{2}}{2 S} \frac{\partial H}{\partial z}+\frac{\partial}{\partial z} \frac{(1+\bar{z} z)^{2}}{2 S} \frac{\partial H}{\partial \bar{z}}\right)\right|_{z=z_{c l}, \bar{z}=\bar{z}_{c l}}
$$

and represents the main subject of our discussion.

In particular, for a Hamiltonian linear in the spin operators

$$
\hat{H}=\mathbf{C} \cdot \hat{\mathbf{s}}=\frac{1}{2} C_{+} \hat{s}_{-}+\frac{1}{2} C_{-} \hat{s}_{+}+C_{3} \hat{s}_{3},
$$

where $C_{ \pm}=C_{1} \pm i C_{2}$, we obtain the spin coherent-state symbol

$$
H(\bar{z}, z)=S \frac{C_{+} z+C_{-} \bar{z}}{1+\bar{z} z}-S C_{3} \frac{1-\bar{z} z}{1+\bar{z} z}
$$

and the Solari-Kochetov phase correction

$$
\frac{1}{2} \phi_{S K}=-\frac{1}{4}\left(C_{+} z_{c l}+C_{-} \bar{z}_{c l}\right)+\frac{1}{2} C_{3}
$$

Another example is the Hamiltonian of the Lipkin-Meshkov-Glick (LMG) model [26]

$$
\hat{H}_{L M G}=\frac{w}{\sqrt{2}(2 S-1)}\left(\hat{s}_{+}^{2}+\hat{s}_{-}^{2}\right)+\frac{S w}{\sqrt{2}}
$$

which is quadratic in the spin operators. In this case we have

$$
H_{L M G}(\bar{z}, z)=\sqrt{2} S w \frac{\bar{z}^{2}+z^{2}}{(1+\bar{z} z)^{2}}+\frac{S w}{\sqrt{2}}
$$

and

$$
\frac{1}{2} \phi_{S K}^{L M G}=-\frac{w}{\sqrt{2}} \frac{\left(\bar{z}^{2}+z^{2}\right)(2+\bar{z} z)}{(1+\bar{z} z)^{2}} .
$$

The latter has been calculated in [8], and its inclusion into the semiclassical propagator has provided the correct result for the tunnel splitting of the ground state in the LMG model.

To complete the presentation of the semiclassical spin propagator, we would also like to mention another quantization scheme which relies on the contravariant symbol $H^{c t r}(\bar{z}, z)$ given by

$$
\hat{H}=\frac{2 S+1}{\pi} \int \frac{d^{2} z}{(1+\bar{z} z)^{2}} H^{c t r}(\bar{z}, z)|z\rangle\langle z| .
$$

As follows from [25], the relation between the covariant and contravariant symbols in the semiclassical limit $S \rightarrow \infty$ reads

$$
H^{\operatorname{cov}}(\bar{z}, z)=\left[1+\Delta+O\left(1 / S^{2}\right)\right] H^{c t r}(\bar{z}, z)
$$


where

$$
\Delta=\frac{(1+\bar{z} z)^{2}}{2 S} \frac{\partial^{2}}{\partial z \partial \bar{z}}
$$

is the Laplace-Beltrami operator acting on the complex projective plane $C P^{1}=S^{2}$ which is a Kähler homogeneous manifold $\mathrm{SU}(2) / \mathrm{U}(1)$. In view of (53), one may convert formula (41) into a form suitable for the quantization by contravariant symbols.

\section{SOLARI-KOCHETOV PHASE FROM HOLSTEIN-PRIMAKOFF REPRESEN- TATION}

Now we would like to derive the Solari-Kochetov phase exploiting the paradigm of the Sec. [II]. For this purpose we employ the Holstein-Primakoff representation [16] for the spin operators

$$
\begin{aligned}
& \hat{s}_{+}=\hat{s}_{1}+i \hat{s}_{2}=\hat{a}^{\dagger} \sqrt{2 S-\hat{a}^{\dagger} \hat{a}}, \\
& \hat{s}_{-}=\hat{s}_{1}-i \hat{s}_{2}=\sqrt{2 S-\hat{a}^{\dagger} \hat{a}} \hat{a}, \\
& \hat{s}_{3}=\hat{a}^{\dagger} \hat{a}-S,
\end{aligned}
$$

in terms of the standard annihilation and creation operators $\hat{a}$ and $\hat{a}^{\dagger}$ with the commutation relation $\left[\hat{a}, \hat{a}^{\dagger}\right]=1$. It is easy to check that the operators (55) satisfy the $\mathrm{su}(2)$ algebra (37) as well as

$$
\frac{1}{2}\left(\hat{s}_{+} \hat{s}_{-}+\hat{s}_{-} \hat{s}_{+}\right)+\hat{s}_{3}^{2}=S(S+1) .
$$

Besides the representation (55), there actually exist another representations of the spin algebra in terms of bosonic operators. For the review of their properties and applications in physics, we refer to [27, 28].

It is also worth mentioning that in Ref. 29], where the instanton picture of spin tunneling in the LMG model was also considered, the sort of the Holstein-Primakoff representation was used in order to obtain the correct ground-state energy splitting. The authors established some heuristic rule which, however, required an adequate interpretation (see Discussion in 29]). Our approach will allow to refine their prescription and to establish the link to the consideration of the LMG model made in [8].

Let us introduce the semiclassical parameter $h=1 /(2 S)$ and define $\hat{A}=\hat{a} \sqrt{h}$ and $\hat{A}^{\dagger}=\hat{a}^{\dagger} \sqrt{h}$, such that

$$
\left[\hat{A}, \hat{A}^{\dagger}\right]=h .
$$


We also define the operators

$$
\begin{aligned}
& \hat{S}_{+}=h \hat{s}_{+}=\hat{A}^{\dagger} \sqrt{1-\hat{A}^{\dagger} \hat{A}}, \\
& \hat{S}_{-}=h \hat{s}_{-}=\sqrt{1-\hat{A}^{\dagger} \hat{A}} \hat{A}, \\
& \hat{S}_{3}=h \hat{s}_{3}=\hat{A}^{\dagger} \hat{A}-\frac{1}{2},
\end{aligned}
$$

which satisfy the commutation relations

$$
\left[\hat{S}_{+}, \hat{S}_{-}\right]=2 h \hat{S}_{3}, \quad\left[\hat{S}_{3}, \hat{S}_{ \pm}\right]= \pm h \hat{S}_{ \pm}
$$

The square root in (58) should be understood as an expansion in a Taylor series

$$
\sqrt{1-x}=1+\sum_{l=1}^{\infty} b_{l} x^{l}
$$

with $x$ replaced by $\hat{A}^{\dagger} \hat{A}$.

One can immediately notice that the operators (58), when expressed through $\hat{A}$ and $\hat{A}^{\dagger}$, do not depend explicitly on $h$, and that (57) is similar to (19). This enables us to apply formally the Moyal formula (14) with the Poisson bracket (20) to the operators (58), replacing everywhere $\hbar$ by $h$. Considering $\alpha$ and $\bar{\alpha}$ to be the "Weyl symbols" of $\hat{A}$ and $\hat{A}^{\dagger}$, respectively, we can thus define the "Weyl symbols" of the operators (58).

First we find

$$
\begin{aligned}
\left(\hat{A}^{\dagger} \hat{A}\right)_{W} & =\bar{\alpha} \alpha-\frac{h}{2}+O\left(h^{2}\right), \\
\left(\hat{A} \hat{A}^{\dagger}\right)_{W} & =\bar{\alpha} \alpha+\frac{h}{2}+O\left(h^{2}\right), \\
\left(\left(\hat{A}^{\dagger} \hat{A}\right)^{l}\right)_{W} & =(\bar{\alpha} \alpha)^{l}-\frac{h l}{2}(\bar{\alpha} \alpha)^{l-1}+O\left(h^{2}\right) .
\end{aligned}
$$

Exploiting the latter relation and the trivial equality

$$
\frac{d}{d x} \sqrt{1-x}=-\frac{1}{2 \sqrt{1-x}}=\sum_{l=1}^{\infty} l b_{l} x^{l-1}
$$

we establish

$$
\left(\sqrt{1-\hat{A}^{\dagger} \hat{A}}\right)_{W}=\sqrt{1-\bar{\alpha} \alpha}+\frac{h}{4} \frac{1}{\sqrt{1-\bar{\alpha} \alpha}}+O\left(h^{2}\right) .
$$

Further use of the Moyal formula (14) leads to the desired definitions

$$
\begin{aligned}
& \left(\hat{S}_{+}\right)_{W}=\left(\hat{A}^{\dagger} \sqrt{1-\hat{A}^{\dagger} \hat{A}}\right)_{W}=\bar{\alpha} \sqrt{1-\bar{\alpha} \alpha}+\frac{h}{2} \frac{\bar{\alpha}}{\sqrt{1-\bar{\alpha} \alpha}}+O\left(h^{2}\right), \\
& \left(\hat{S}_{-}\right)_{W}=\left(\sqrt{1-\hat{A}^{\dagger} \hat{A}} \hat{A}\right)_{W}=\alpha \sqrt{1-\bar{\alpha} \alpha}+\frac{h}{2} \frac{\alpha}{\sqrt{1-\bar{\alpha} \alpha}}+O\left(h^{2}\right), \\
& \left(\hat{S}_{3}\right)_{W}=\left(\hat{A}^{\dagger} \hat{A}-\frac{1}{2}\right)_{W}=\bar{\alpha} \alpha-\frac{1}{2}-\frac{h}{2}+O\left(h^{2}\right) .
\end{aligned}
$$


We can construct the approximate realization of the $\mathrm{su}(2)$ algebra with respect to the Poisson bracket (20). Taking into account (15) and the commutation relations (59) we deduce that

$$
\begin{aligned}
& i\left\{\left(\hat{S}_{+}\right)_{W},\left(\hat{S}_{-}\right)_{W}\right\}_{\bar{\alpha}, \alpha}=2\left(\hat{S}_{3}\right)_{W}+O\left(h^{2}\right), \\
& i\left\{\left(\hat{S}_{3}\right)_{W},\left(\hat{S}_{ \pm}\right)_{W}\right\}_{\bar{\alpha}, \alpha}= \pm\left(\hat{S}_{ \pm}\right)_{W}+O\left(h^{2}\right) .
\end{aligned}
$$

These formulae can be checked by straightforward calculation using (666).

There exists, however, a subtlety that should be spelled out here. The spin operators (58) act in the finite Hilbert space in contrast to the operators $\hat{q}, \hat{p}$ and $\hat{H}(\hat{q}, \hat{p})$ in the flat case which act in a different - infinite - Hilbert space. Nevertheless, the "Weyl symbols" (66) of the operators (58) do make sense locally in the semiclassical limit $h \rightarrow 0$, and, as we shall see, reproduce the Solari-Kochetov phase. Similarly to the flat case, we are going to recognize it for a Hamiltonian linear in the spin operators

$$
\hat{H}=\frac{1}{h} \mathbf{C} \cdot \hat{\mathbf{S}}=\frac{1}{h}\left[\frac{1}{2} C_{+} \hat{S}_{-}+\frac{1}{2} C_{-} \hat{S}_{+}+C_{3} \hat{S}_{3}\right]
$$

in the difference between its principal and "Weyl" symbols

$$
H(\bar{\alpha}, \alpha)-H_{W}(\bar{\alpha}, \alpha)=\delta H(\bar{\alpha}, \alpha)+O(h) .
$$

(There is a small distinction in notations in comparison with the flat case since the classical action and the naive classical Hamiltonian for spin are already divided by the semiclassical parameter $h$ and therefore $H(\bar{\alpha}, \alpha)$ and $\delta H(\bar{\alpha}, \alpha)$ are of order $O\left(h^{-1}\right)$ and $O(1)$, respectively.)

According to (66) the principal symbol and the next-order correction of (69) are given, respectively, by

$$
\begin{aligned}
H(\bar{\alpha}, \alpha) & =\frac{1}{h}\left[\frac{1}{2} C_{+} \alpha \sqrt{1-\bar{\alpha} \alpha}+\frac{1}{2} C_{-} \bar{\alpha} \sqrt{1-\bar{\alpha} \alpha}+C_{3}\left(\bar{\alpha} \alpha-\frac{1}{2}\right)\right], \\
\delta H(\bar{\alpha}, \alpha) & =-\frac{C_{+} \alpha+C_{-} \bar{\alpha}}{4 \sqrt{1-\bar{\alpha} \alpha}}+\frac{1}{2} C_{3} .
\end{aligned}
$$

To compare these expressions with (47) and (48), respectively, we use the Darboux transformation

$$
z=\frac{\alpha}{\sqrt{1-\bar{\alpha} \alpha}}, \quad \bar{z}=\frac{\bar{\alpha}}{\sqrt{1-\bar{\alpha} \alpha}} .
$$

It makes the Kähler symplectic structure locally flat and converts the classical equations of motion (44) into

$$
\dot{\alpha}=-i h \frac{\partial H(\bar{\alpha}, \alpha)}{\partial \bar{\alpha}}+O(h), \quad \dot{\bar{\alpha}}=i h \frac{\partial H(\bar{\alpha}, \alpha)}{\partial \alpha}+O(h) .
$$


The terms $O(h)$ appear due to the finiteness of the $(\alpha, \bar{\alpha})$ phase space which is a disc on the complex plane. However, they become negligible as $h \rightarrow 0$. All the other terms in (74) have the order $O(1)$.

Thus, after the transformation (73) we observe the coincidence of the principal symbols (47) and (71) and obtain the desired relation

$$
\frac{1}{2} \phi_{S K}=\delta H
$$

Our consideration is not restricted to the case of Hamiltonians linear in spin operators. The formula (75) can be proved valid when the Hamiltonian is a more general element of the enveloping algebra (i.e. a polynomial in spin operators).

Let us consider, for example, the Hamiltonian

$$
\hat{H}=c \cdot \frac{(2 S-n) !}{(2 S-1) !}\left(\hat{s}_{+}^{n}+\hat{s}_{-}^{n}\right),
$$

where $n$ is an arbitrary integer number. For $n=2$ and $c=w / \sqrt{2}$ it coincides (up to the constant factor) with the Hamiltonian of the LMG model (49).

We note that it is important to introduce the $S$-dependent coefficient in (76) so that to make the respective covariant symbol proportional to $S$ :

$$
H^{c o v}=c \cdot 2 S \frac{\bar{z}^{n}+z^{n}}{(1+\bar{z} z)^{n}}
$$

This allows to identify (777) with the principal symbol, or classical Hamiltonian, which contains the leading-in- $S$ term only.

First, we calculate the Solari-Kochetov phase according to the original formula (45), and obtain

$$
\frac{1}{2} \phi_{S K}=-c \cdot \frac{n}{2} \frac{\left(\bar{z}^{n}+z^{n}\right)(n+\bar{z} z)}{(1+\bar{z} z)^{n}} .
$$

Now we would like to show that the same expression can be obtained from (75) using the Holstein-Primakoff representation. We rewrite the Hamiltonian (76) in terms of the operators (58)

$$
\hat{H}=c \cdot h^{-n} \frac{\left(h^{-1}-n\right) !}{\left(h^{-1}-1\right) !}\left(\hat{S}_{+}^{n}+\hat{S}_{-}^{n}\right),
$$

and find its "Weyl" symbol

$$
\begin{aligned}
(\hat{H})_{W} & =c \cdot \frac{1}{h}\left(1+\frac{h n(n-1)}{2}\right)\left[\frac{\bar{z}^{n}+z^{n}}{(1+\bar{z} z)^{n}}+\frac{h n}{2} \frac{\bar{z}^{n}+z^{n}}{(1+\bar{z} z)^{n-1}}\right]+O(h) \\
& =c \cdot\left[\frac{1}{h} \frac{\bar{z}^{n}+z^{n}}{(1+\bar{z} z)^{n}}+\frac{n}{2} \frac{\left(\bar{z}^{n}+z^{n}\right)(n+\bar{z} z)}{(1+\bar{z} z)^{n}}\right]+O(h) .
\end{aligned}
$$


This expression is derived due to

$$
\begin{aligned}
\left(\hat{S}_{+}^{n}\right)_{W} & =(\bar{\alpha} \sqrt{1-\bar{\alpha} \alpha})^{n}+\frac{h n}{2} \bar{\alpha}^{n}(\sqrt{1-\bar{\alpha} \alpha})^{n-2}+O\left(h^{2}\right) \\
& =\frac{\bar{z}^{n}}{(1+\bar{z} z)^{n}}+\frac{h n}{2} \frac{\bar{z}^{n}}{(1+\bar{z} z)^{n-1}}+O\left(h^{2}\right), \\
\left(\hat{S}_{-}^{n}\right)_{W} & =(\alpha \sqrt{1-\bar{\alpha} \alpha})^{n}+\frac{h n}{2} \alpha^{n}(\sqrt{1-\bar{\alpha} \alpha})^{n-2}+O\left(h^{2}\right) \\
& =\frac{z^{n}}{(1+\bar{z} z)^{n}}+\frac{h n}{2} \frac{z^{n}}{(1+\bar{z} z)^{n-1}}+O\left(h^{2}\right),
\end{aligned}
$$

and

$$
\frac{(N-n) !}{N !}=N^{-n}\left(1+\frac{1}{N} \frac{n(n-1)}{2}+O\left(\frac{1}{N^{2}}\right)\right),
$$

for $N \equiv 2 S=h^{-1}$. The relations (73) have been also employed.

Thus, we see that the leading term in (801) coincides with (77), and the next-to-leading term is in agreement with (78). We note that in our derivation of the Solari-Kochetov phase it was important to expand in a series of $h$ the $h$-dependent coefficient - the ratio of two factorials - which was inherited from the quantum Hamiltonian (79)).

\section{DISCUSSION}

We put forward a proposition to determine the extra-phase correction in the semiclassical expression for a propagator as the difference between the principal and the Weyl symbol of the quantum Hamiltonian. Based on the Wigner-Weyl calculus, it becomes a well-defined and efficient computational prescription.

We offered to exploit this paradigm for the case of the spin propagator, making use of the Holstein-Primakoff representation of the $\mathrm{su}(2)$ algebra. However, there exists a subtle issue concerning the finiteness of the spin Hilbert space. It also shows up on the classical level: the classical phase space in such a representation is a flat disc which has finite volume. Nevertheless, in the semiclassical limit $S \rightarrow \infty$ the difference between the principal and the "Weyl" symbols appears to be well-defined. This is also confirmed by the possibility to construct the $\mathrm{su}(2)$ algebra realization in terms of the Poisson bracket $i\{\cdot, \cdot\}_{\bar{\alpha}, \alpha}$ with the required accuracy (modulo terms $O\left(h^{2}\right)$ ). Obviously, this amounts to defining the spin Weyl symbols only locally.

We applied the developed prescription for the calculation of the phase correction to the semiclassical spin coherent-state propagator for systems with the Hamiltonian which is either 
linear or nonlinear in spin operators. The presented consideration can be straightforwardly generalized for any Hamiltonian which is a polynomial in spin operators. Our prescription is rather simple from a computational point of view, and it does not require the use of the polynomial tensor operators (cf. [14]).

In summary, we would like to motivate the usefulness of the "Weyl" symbol for spin with the following reference. The idea to introduce such symbol is employed in order to reveal the similarity between the phase corrections to the semiclassical flat and spin propagators, and it appears quite naturally when one considers the semiclassical limit of the HolsteinPrimakoff (HP) representation including the next-to-leading term. In Ref. 29] the LipkinMeshkov-Glick (LMG) model (quadratic in spin) has been considered using the sort of the HP representation. The authors recognized the necessity of the phase correction to the semiclassical spin propagator within their approach and formulated the heuristic rule for its calculation. However, they stated the lack of its adequate interpretation. On the other hand, the consideration of the LMG model made in Ref. 8] uses the original Solari-Kochetov expression for the phase correction. The relation between these two approaches is missing, although both of them have led to the correct result. Thus, introducing the "Weyl" symbol for spin helps to bridge the gap between two different interpretations of the same extra-phase correction.

\section{ACKNOWLEDGEMENTS}

The author would like to thank Evgueny Kochetov for many stimulating discussions, critical reading of the manuscript and suggestions that helped to improve the presentation considerably. Matthias Brack, Hajo Leschke, Simone Warzel and Oleg Zaitsev are acknowledged for their useful comments. The work has been supported by the Deutsche Forschunsgemeinschaft and by a travel grant of the Heisenberg-Landau program.

[1] E. M. Chudnovsky and L. Gunther, Phys. Rev. Lett. 60, 661 (1988).

[2] D. Loss, D. P. Vincenzo, and G. Grinstein, Phys. Rev. Lett. 69, 3232 (1992).

[3] J. von Delft and C. L. Henley, Phys. Rev. Lett. 69, 3236 (1992).

[4] A. Garg, Europhys. Lett. 22, 205 (1993). 
[5] M. Stone, K-S. Park, and A. Garg, J. Math. Phys. 41, 8025 (2000).

[6] H. Kuratsuji and Y. Mizobuchi, J. Math. Phys. 22, 757 (1981).

[7] A. Garg and G-H. Kim, Phys. Rev. B 45, 921 (1992).

[8] A. Garg, E. Kochetov, K-S. Park, and M. Stone, J. Math. Phys. 44, 48 (2003).

[9] H. Solari, J. Math. Phys. 28, 1097 (1987).

[10] E. Kochetov, J. Math. Phys. 36, 4667 (1995).

[11] V. R. Vieira and P. D. Sacramento, Nucl. Phys. B 448, 331 (1995).

[12] M. Pletyukhov and O. Zaitsev, J. Phys. A: Math. Gen. 36, 5181 (2003).

[13] M. C. Gutzwiller, J. Math. Phys. 12, 343 (1971).

[14] A. Garg and M. Stone, Phys. Rev. Lett. (in print); cond-mat/0304125.

[15] J. E. Moyal, Proc. Cambridge Philos. Soc. 45, 99 (1949).

[16] T. Holstein and H. Primakoff, Phys. Rev. 58, 1098 (1940).

[17] A. A. Kirillov, Geometric Quantization, in Dynamical Systems IV, ed. V.I. Arnold (Springer, Berlin, 1988), p. 137.

[18] M. Stone, Nucl. Phys. B 314, 557 (1989).

[19] J. H. van Vleck, Proc. Natl. Acad. Sci. USA 14, 178 (1928).

[20] M. C. Gutzwiller, J. Math. Phys. 8, 1979 (1967).

[21] E. Kochetov, J. Phys. A: Math. Gen. 31, 4473 (1998).

[22] F. A. Berezin, Sov. Phys. Usp. 23, 763 (1980).

[23] K. E. Cahill and R. J. Glauber, Phys. Rev. 177, 1857 (1969).

[24] A. Voros, Phys. Rev. A 40, 6814 (1989).

[25] F. A. Berezin, Math. USSR (Izvestia) 8, 1109 (1974).

[26] H. J. Lipkin, N. Meshkov, and A. J. Glick, Nucl. Phys. 62, 188 (1965).

[27] J. P. Blaizot and E. R. Marshalek, Nucl. Phys. A 309, 422 (1978); 309, 453 (1978).

[28] P. Garbaczewski, Phys. Rep. 36, 65 (1978).

[29] V. I. Belinicher, C. Providencia, and J. da Providencia, J. Phys. A: Math. Gen. 30, 5633 (1997). 